2015-04-01

\title{
Optical Diagnostics - Spectropathology for the Next Generation
}

\author{
Malgorzata Baranska \\ Jagiellonian University Cracow \\ Hugh Byrne \\ Technological University Dublin, hugh.byrne@tudublin.ie
}

Follow this and additional works at: https://arrow.tudublin.ie/biophonart

Part of the Analytical, Diagnostic and Therapeutic Techniques and Equipment Commons, and the Physics Commons

\section{Recommended Citation}

"Optical diagnostics - spectropathology for the next generation", Malgorzata Baranska and Hugh J. Byrne, Analyst, Editorial, Special Issue "Optical Diagnostics", 140, 2064-2065 (2015) doi:10.1039/ c5an90024g

This Article is brought to you for free and open access by the DIT Biophotonics and Imaging at ARROW@TU Dublin. It has been accepted for inclusion in Articles by an authorized administrator of ARROW@TU Dublin. For more information, please contact arrow.admin@tudublin.ie, aisling.coyne@tudublin.ie,gerard.connolly@tudublin.ie.

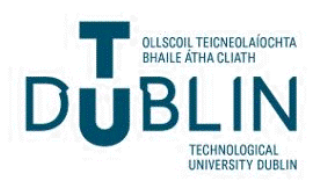




\section{Analyst}

\section{EDITORIAL}

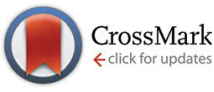

Cite this: Analyst, 2015, 140, 2064

DOI: $10.1039 / \mathrm{c} 5 \mathrm{an} 90024 \mathrm{~g}$

www.rsc.org/analyst

\section{Optical diagnostics - spectropathology for the next generation}

This latest Optical Diagnosis themed issue of Analyst, guest-edited by Prof. Malgorzata Baranska, Jagiellonian University, Krakow, Poland, and Prof. Hugh J. Byrne, Dublin Institute of Technology, Ireland, is drawn from the participants and proceedings of the International conference, SPEC 2014: Shedding New Light on Disease, which was held on the 17th-22nd August 2014 in Krakow, Poland. The conference was co-chaired by Prof. Baranska, Prof. Byrne and Prof. Anna Sulkowska, Medical University of Silesia, Katowice, Poland.

The event was the eighth in the series of biennial conferences, the aim of which is to bring together clinicians and scientists who have joined forces in the quest for novel biomedical applications of infrared and Raman spectroscopy to improve patient care. Recent advances in the biological sciences and medicine have led to an increasing demand for real-time and minimally invasive chemical and structural information on biological materials. Due to its unique fingerprinting capability, vibrational spectroscopy plays a significant role in histopathology, cytology, biopsy targeting, surgical targets, treatment-monitoring and drug studies.

The conference aimed to highlight further advances in state of the art and emerging biomedical applications of vibrational spectroscopy, while reviewing the challenges in the context of other emerging technologies. The programme was constructed in an attempt to prioritise real-world applications from the outset, systematically progressing from research towards in-vivo, ex-vivo and in-vitro applications, as well as emerging technologies and data processing.

A feature of the conference was the daily discussion sessions which were aimed at a critical assessment of the routes towards the further development of clinical applications, and were moderated by members of the SPEC International Advisory Board. The deliberations are summarised in the second editorial 'Spectropathology for the next generation: Quo vadis?' (Byrne et al., c4an02036g), under the headings of (i) Translational research into in-vivo clinical applications, (ii) Ex-vivo tissue biopsies, body fluids and cytological samples for diagnostics and disease studies, and (iii) In-vitro cell culture and 3D models for research and medical applications. A number of critical concerns are highlighted under the respective headings, including (i) the need for the engagement with the clinical community and adherence to clinical standards in terms of statistical relevance, (ii) standardisation of protocols for sample preparation, presentation and measurement and data processing, and (iii) continued development of instrumentation and models to explore the limitations of the techniques.

The scope of the conference and the contents of this themed issue are indicative of the progress in the understanding of the complexity of spectroscopic characterisation of biological materials, and data preprocessing and postprocessing methods can increasingly be applied with confidence to give true biochemical representations of tissue, cells and bodily fluids. The issue contains a total of 51 contributed communications and articles, drawing from research activities across the globe, including Australia, India, Japan, US and across Europe with a notably strong representation from the host country, Poland.

The increased development of fibre probes shows great promise for in-vivo diagnosis and intra-operative applications, and although Raman probes operating at visible or near-infrared wavelengths - are relatively well established, probes for FTIR spectroscopy are less so. The study by Padalkar and Pleshko (c4an01987c) details the development of such probes for the in-vivo analysis of cartilage.

For clinical applications, the research field remains dominated by ex-vivo applications of tissue, cells and biofluids. Spectrohistopathological studies using either FTIR or Raman profiling range from analysis of cancer markers in adrenal glands (Dudala et al., c4an01891e), salivary tissue (BrozekPluska et al., c4an01394h), lung tumour (Gerwert et al., c4an01978d), breast tissue (Abramczyk et al., c4an01876a; Brozek-Pluska et al., c4an01877j), and primary and metastatic melanoma (Wald and Goormaghtigh, c4an01831a). Further studies include analysis of the pituitary gland (Banas et al., c4an01985g), calcification of the aortic valve (Kaczor et al., c4an01856g), the vitamin A content in murine lung and liver tissue (Marzec et al., c4an01881h; Baranska et al., c4an01878h) and endothelial function/dysfunction (Kaczor et al., c4an01870b; Rygula et al., c4an01998a). Animal models also feature strongly in 
the studies of Chwiej et al. (c4an01857e) as epileptic models, and as obesity models in the work of Severcan et al. (c4an02008a).

Cytological studies, using FTIR and FTIR-ATR spectroscopy also feature strongly, in, for example, the study by Diem et al. (c4an01884b) of oesophageal cells. Abramczyk et al. (c4an01875c) demonstrate the role of lipid droplets and adipocytes in cancer by comparing the Raman spectral profiles of cell cultures to those of adipocytes in cancerous human breast tissue.

The contents of this issue reflect an increasing prominence of studies of blood cells (Wood et al., c4an01904k) and peripheral blood lymphocytes (Goormaghtigh et al., c4an01855a and c4an02247e), and indeed of bodily fluids themselves, using both FTIR and Raman spectroscopy, notably blood plasma (Štovíčková et al., c4an01874e; Malek et al., c4an01864h; Garnotel et al., c4an01942c; Tatarkovič et al., c4an01880j) and serum (Chilakapati et al., c4an01860e), potentially promising more accessible routine screening applications of vibrational spectroscopy.

Spectroscopic techniques remain a powerful tool for basic research and may find applications in vitro for toxicology and drug screening, as exemplified by the study of cellular uptake of doxorubicin by Chlopicki, Baranska and coworkers (c4an01882f), and the study of the biocompatibility of nanocomposites by Wesełucha-Birczyńska et al. (c4an02284j), Malek et al. (c4an01988a), and Campbell et al. (c4an02365j), demonstrate the use of SERS-based nanosensors to monitor the intracellular $\mathrm{pH}$ in endothelial cells. Equally, such in-vitro studies can provide valuable explorations for new 3D cell culture models (Smolina and Goormaghtigh, c4an01833h), developing neural systems (Sato et al., c4an01961j) and disease models (malaria) (Hobro, Smith and coworkers, c4an01850h). Mosig et al. (c4an02153c) demonstrate the colocalization of fluorescence and Raman microscopic images for the identification of subcellular compartments, and thus the potential of the technique for in-vitro screening.

Although the proof of concept of a range of potential clinical applications of vibrational spectroscopy has been well demonstrated, there remains the need for standardisation of measurement protocols and data processing, as highlighted in the discussion editorial (Byrne et al., c4an02036g). Exploration of the impact of tissue processing (Stone et al., c4an02122c; Wood et al., c4an02034k) as well as measurement configurations for FTIR spectroscopy (Gardner et al., c4an01975j; Mignolet and Goormaghtigh, c4an01834f; Wood et al., c4an01901f; Malek et al., c4an01842g) feature strongly. Gardner et al. (c4an02053g) also propose protocols for enhanced FTIR bench-top imaging of single biological cells. The relative merit of different classification and data-mining algorithms continues to be a subject for investigation (Wood et al., c4an01783h;
Surowka et al., c4an01867b; Gobinet et al., c4an01937g; Diem et al., c4an01832j and c4an01879f; Meade et al., c4an01887g), while Keating et al. (c4an02167c) demonstrate the importance of simulated datasets for method validation.

Emerging technologies continue to push the performances in terms of spatial resolution (Gough et al., c4an01982b), sampling depth (Ariese et al., c4an01889c) and the emergence of quantum cascade lasers as IR sources promises increased scanning speeds for large area spectral mapping (Kröger-Lui et al., c4an02001d). Meanwhile, Czepiel, Wesełucha-Birczyńska and co-workers (c4an01947d) give glimpses into applications beyond human pathologies.

The field of clinical applications of vibrational spectroscopy continues to engage a broad interdisciplinary research community across the world, including spectroscopists, chemists, biochemists etc. The field is furthermore becoming increasingly intersectoral, as instrument companies address the challenge of faster acquisition speeds over larger areas, and the medical community itself becomes more 'spectrophilic' and engaged in promoting the potential of spectropathology for the next generation. We hope you find the articles gathered in this themed issue of Analyst stimulating and inspiring.

Malgosia Baranska

Jagiellonian University, Krakow, Poland Hugh J. Byrne

Dublin Institute of Technology, Ireland 